\title{
Direct numerical simulation (DNS) investigation of turbulent open channel flow of a Herschel-Bulkley fluid
}

\author{
R. Guang Rheology and Materials Processing Centre, RMIT University, Australia
}

M. Rudman CSIRO Mathematical and Information Sciences, Australia

A. Chryss CSIRO Process Science and Engineering, Australia

P.T. Slatter Rheology and Materials Processing Centre, RMIT University, Australia

S. Bhattacharya Rheology and Materials Processing Centre, RMIT University, Australia

\begin{abstract}
In this research, the turbulent behaviour of non-Newtonian suspensions in open channel conditions will be examined and investigated. With lack of fundamental understanding about the mechanisms involved about how turbulent flows of non-Newtonian fluid transport suspension particles, direct numerical simulation may come into the research as a useful validation tool. A better understanding of the mechanism operating in the turbulent flow of non-Newtonian suspensions in open channel flow would lead to improved design of many of the systems used in the mining and mineral processing industries. The main aim of the study is to describe how does a non-Newtonian fluid transport particles in an open channel and validation of a computational model.

Direct numerical simulation (DNS) of the turbulent flow of non-Newtonian fluids in an open channel is modelled using a spectral element-Fourier method. The simulation of a yield-pseudoplastic fluid using the Herschel-Bulkley model agrees qualitatively with experimental results from field measurements of mineral tailing slurries. The effect of variation in flow behaviour index is investigated and used to assess the sensitivity of the flow to these physical parameters. This methodology is seen to be useful in designing and optimising the transport of slurries in open channels.
\end{abstract}

\section{Introduction}

When self-formed channels flow at a sufficient gradient or slope, they can generate a certain level of turbulence. Therefore open channels used to transport ore slurries and tailings streams can be a more economic alternative to pumping when terrain allows. Despite years of research, at the present the design of these flumes is often done using crude estimates based on the conditions established for water with a limited set of field observations (Wilson, 1991).

Steep self-formed channels flow at a sufficient gradient to generate a level of turbulence that is able to maintain all the tailings particles in suspension. However, with a shallower gradient, the turbulence intensity reduces, and more solids settle in the channel. It is known that the turbulent coherent structures are likely to contribute to the mechanics of suspending particles away from the channel bed (Verbanck, 2000). Yet, there is a lack of fundamental understanding about many of the mechanisms involved in how a turbulent flow of a non-Newtonian carrier fluid maintains particles in suspension.

Experimental measurement of velocity profiles and turbulence statistics can be used to determine the state of channel flow. If the rheology of the suspension is known, then computational techniques can be used to simulate the flow patterns. The main benefit of using a DNS technique is that once it is validated, it can be used to model flow behaviour and provide a detailed description of the turbulent structure which can then be applied to particle transportation in channels.

Earlier work, Kim et al. (1987) have simulated turbulent channel flow of Newtonian fluids with Reynolds number up to 3,300. There have been some DNS of the turbulent flow of polymer solutions as well (Sureskumar et al., 1997). The study considered the drag reduction that arises in dilute polymer solutions in which shear-thinning behaviour was unimportant. Dimitropoulos et al. (2005) have also used DNS to 
simulate viscoelastic turbulent boundary layer flow. It was found that as drag reduction increases, the streamwise velocity fluctuations are decreased. Furthermore, Rudman and Blackburn $(2003,2006)$ have used spectral element method to simulate non-Newtonian flow in pipes. The velocity distribution resulting from the present approach of adopting the Herschel-Bulkley model showed good agreement in terms of shape and magnitude when compared with the experimental data. The objective of this paper is to present some interesting findings from a study undertaken for time independent shear-thinning viscoplastic fluids in an open channel flow, whose rheology can be described using the Herschel-Bulkley model. The study of nonNewtonian suspensions in open channels will provide the additional fundamental information for understanding the behaviour of self-formed channels in situations such as centrally thickened discharged (CTD) stack. The same information will also be applicable to the design and operation of industrial flumes for the transport of mineral suspensions with expected advantages in management and economic outcomes.

\subsection{Rheological models}

In the current work, the viscosity $\eta$ of the fluid can be described using the Herschel-Bulkley rheological model:

$$
\eta=\frac{\tau_{Y}}{\not \&}+K \not \mathbb{Z}^{-1}
$$

Where: $K$ is the consistency index, $n$ is the flow behaviour index and $\tau_{y}$ is the fluid yield stress.

\subsection{Wall viscosity}

When the viscosity varies in space and time, the appropriate viscosity scale to use in order to define a Reynolds number is not obvious. Therefore in this paper, the Reynolds number is defined by mean wall viscosity. It is calculated from the mean wall shear stress, $\tau_{\mathrm{w}}$. It is determined directly from the applied axial pressure gradient.

$$
\tau_{w}=\frac{\delta p}{\delta z} \rho \frac{A}{C}
$$

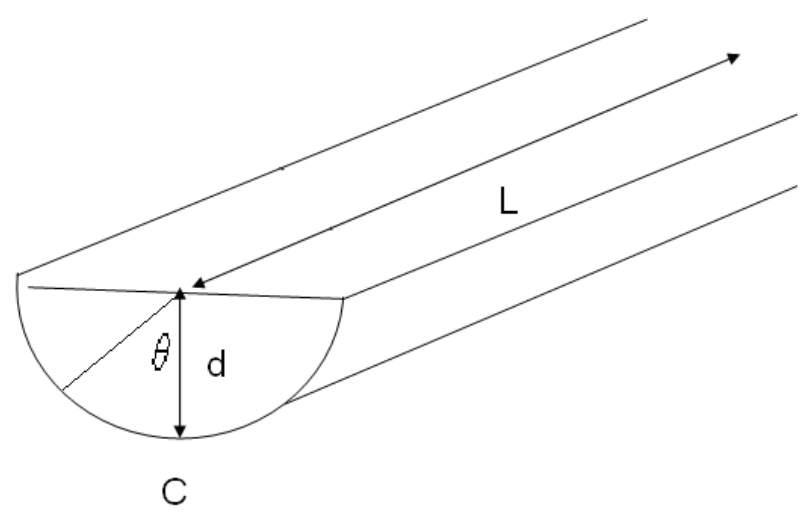

\section{Figure 1 Simulation channel geometry}

Where:

$$
C=R \theta
$$

$A$ is cross section area. Assuming a Herschel-Bulkley rheology,

$$
\eta_{w}=K^{1 / n} \frac{\tau_{w}}{\left(\tau_{w}-\tau_{y}\right)^{1 / n}}
$$




\subsection{Wall units}

Wall units are introduced with the wall viscosity replacing the Newtonian viscosity. Therefore the friction velocity is defined as $U^{*}=\sqrt{\tau_{w} / \rho}$, the non-dimensional velocity is $U^{+}=U / U^{*}$ and the non-dimensional distance from the wall is written $y^{+}=\left(\rho U^{*} / \eta_{w}\right) y$.

\section{Experimental method}

The previous experimental campaign was taken by former PhD student, Tim Fitton in 2004 (Fitton, 2007). The experiment was conducted at the Sunrise Dam Gold Mine, Western Australia. The experimental set up consists of a flume channel of $340 \mathrm{~mm}$ internal diameter with semi-circular cross section. The tailings slurry was supplied to the plunge box which was located at the upstream end through a $150 \mathrm{~mm}$ high density polyethylene (HDPE) pipe with an outlet $20 \mathrm{~cm}$ above the plunge box floor. A diaphragm valve was installed in the pipe to adjust the flow rate of the tailing. Further details of this experiment and associated instrumentation are given in Fitton (2007).

Rheometric analysis was performed on samples of the slurries at different concentrations to determine its rheological parameters. This enables the fitting of a Herschel-Bulkley rheological model. Rheological parameters based on the experiment data were used in the simulation. The modelling presented in the next section has been compared with experimental work presented by Fitton (2007).

\section{$3 \quad$ Numerical method}

The numerical method is based on spectral element/Fourier formulation. The three-dimensional (3D) spatial discretisation uses isoparametrically mapped quadrilateral spectral elements in the cross section $(\mathrm{x}, \mathrm{y})$ that allows arbitrary geometry in the (x,y) plane, and $\mathrm{z}$ (out-of-plane) direction. The z-axis is aligned with the flow direction and uses a Fourier expansion that results in a periodic geometry in this direction. Details of numerical method may be found in Rudman and Blackburn (2006).

In order to drive the flow in the axial (z) direction, a body force per unit mass equivalent to the pressure gradient measured in the experiments is applied to the z-momentum equation. This approach allows the pressure to be periodic in the axial direction.

The computational domain consists of $51 \times 15$ th order elements in the channel cross section and 384 Fourier modes in the axial direction, with domain lengths of $0.5 \pi \mathrm{D}$. The reason for choosing a small domain length is because with a longer domain length and current number of planes (384) the simulation time would be too great. A larger number of $\mathrm{z}$ planes can increase the resolution of the velocity contour. Therefore the domain length has been shortened to meet the demand of the large number of $\mathrm{z}$ planes. This is the summary of simulation parameter. 
Table 1 Parameters for simulation

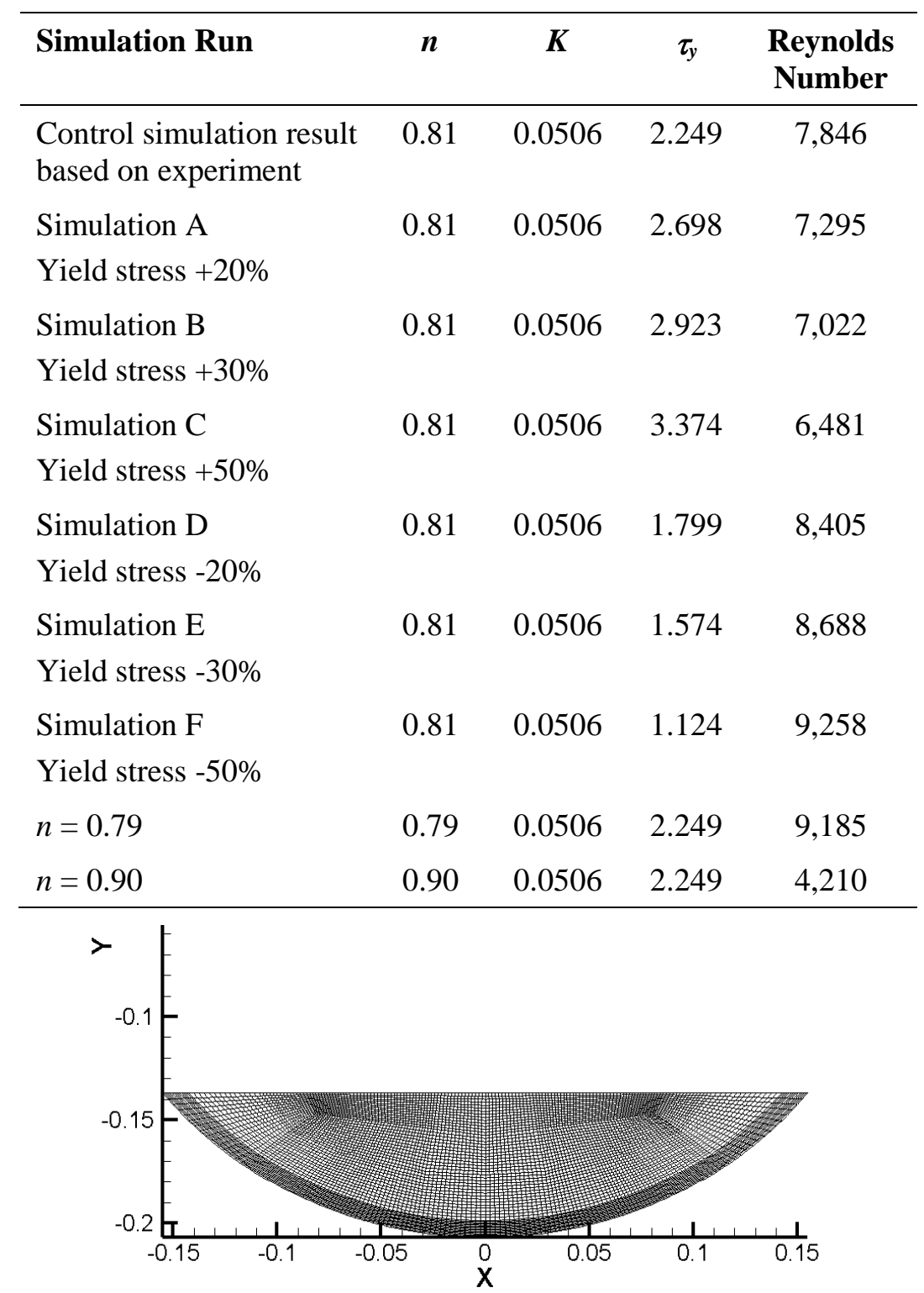

Figure 2 Simulation mesh

\subsection{Boundary conditions}

In this simulation, boundary conditions can be only of Dirichlet or Neumann type. Therefore the wall region has no-slip condition, and at the free surface it is forced to have a stress free surface condition.

\section{$4 \quad$ Experimental results}

The transport characteristics of the slurry were measured. The generalised Reynolds number is based on a wall viscosity that is calculated by wall shear stress.

Clapp (1961) reported the results of experimental measurements of the turbulent pipe flow of power law fluids with flow behaviour indices in the range of 0.698-0.813. Clapp determined that the logarithmic velocity profile for the turbulent flow of power law fluids is a function of the flow index, $n$, and satisfies:

$$
\hat{U}=\frac{A}{n}+\frac{B}{n} \ln \hat{y}
$$


Where:

$$
\hat{y}=\left[\frac{\left(\rho^{n} \tau_{w}{ }^{2-n}\right)^{1 / 2}}{K}\right] y^{n}
$$

The value of these coefficients for well-developed turbulent flow of Newtonian fluids (where $n=0$ ) are now generally accepted to be $\mathrm{A}=5.5, \mathrm{~B}=2.5$. However in this case, the logarithmic profile used for all plots is:

$$
U^{+}=5.5+2.5 \ln y^{+}
$$

The mean axial velocity data for the slurry in the central plane is presented in Figure 3, in wall units, together with the logarithmic velocity profile. The experimentally measured velocity profile has a linear relationship between $\mathrm{U}^{+}$and $y^{+}$in the near wall region. In the logarithmic region, the actual velocity profile for the slurry is slightly higher than the logarithmic velocity profile. At the free surface region, the measured velocity profile declined after a maximum. According to Joung et al. (2007) and Nezu (2005), this could be concluded as a secondary flow effect where the lower velocity/momentum material is advected into the otherwise high velocity/momentum region. Therefore there is a dip in the axial velocity profile after maximum velocity has been reached. This happens regularly in non circular channel flow. It is also suggested by Nezu and Nakagawa (1993) that this phenomenon be called the 'velocity-dip', and it is peculiar to open channel flows. The bulk velocity of the experimental result is $1.06 \mathrm{~m} / \mathrm{s}$.

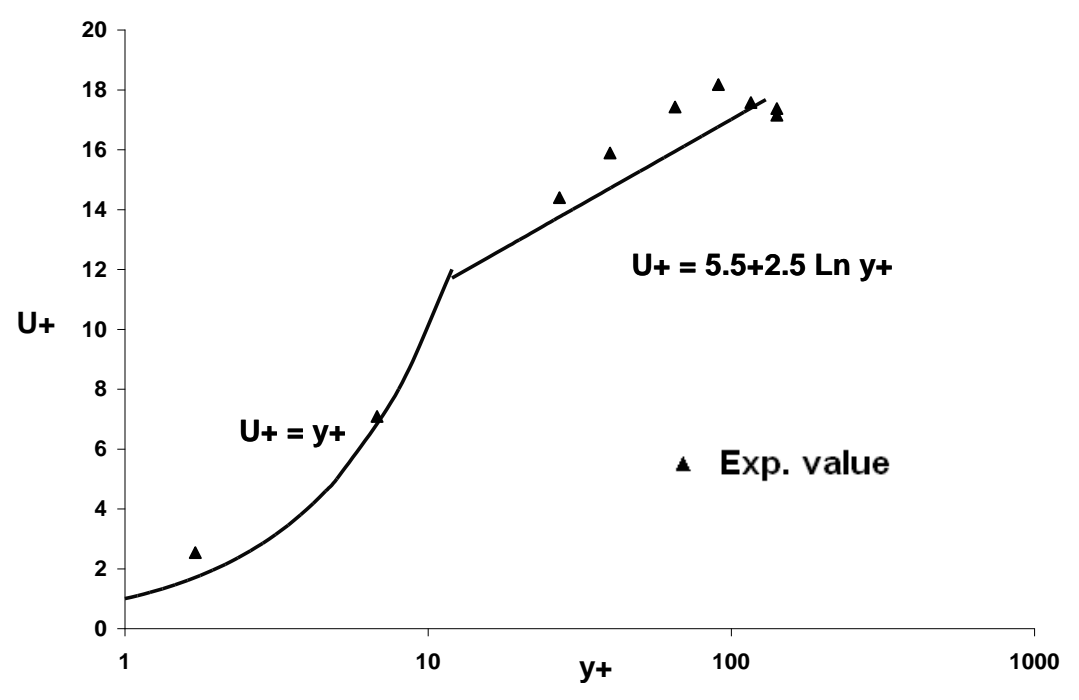

Figure 3 Experimentally measured velocity profile for slurry

\subsection{Discussion of results}

The computationally predicted profile for the control simulation at Reynolds number $=8,294$ is presented in Figure 4. DNS turbulent pipe flow at Reynolds number $=7,500$ flow data from Rudman and Blackburn (2006) is also presented in the same plot. The velocities have been non-dimensionalised. The nondimensionalisation is undertaken using the wall viscosity give in Equation (2). The channel profile is also in good agreement with accepted profile for turbulent pipe flow. All profiles have a linear relationship between $\mathrm{U}^{+}$and $y^{+}$in the near wall region. In the logarithmic region, the simulation profile and Rudman and Blackburn (2006) profile are above the Newtonian logarithmic velocity profile. This is consistent with experimental results. However, Rudman and Blackburn's (2006) profile used a power law fluid rather than a Herschel-Bulkley fluid. 


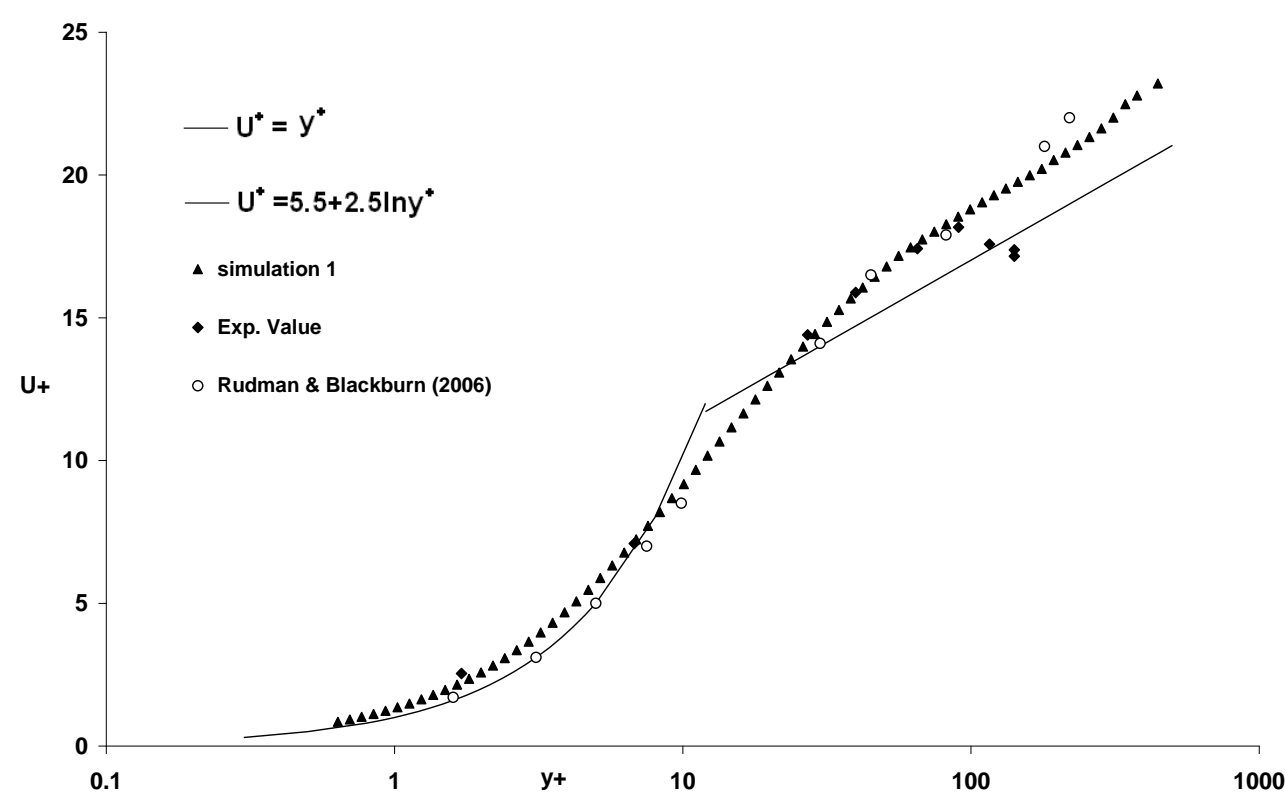

Figure 4 Experimentally measured velocity profile in conventional wall units for slurry in comparison of simulation results

The bulk velocity of the simulation is $1.48 \mathrm{~m} / \mathrm{s}$ where as the bulk velocity of the experimental result is $1.06 \mathrm{~m} / \mathrm{s}$. It is seen that a significant disagreement between simulation and measurement exists. Not only is the predicted velocity is approximately $39 \%$ higher than experimental, but the velocity profile has somewhat different shape to the experimental profiles. The simulation profile and experimental profile have the same magnitude in the near wall region.

\section{$5 \quad$ Numerical results}

The results from six simulations are presented in Table 1. Simulation A, B, C have yield stresses increased by 20,30 and $50 \%$ of the control value of $2.249 \mathrm{~Pa}$ respectively. Simulation D, E, F have yield stresses decreased of the control value by 20,30 and $50 \%$ respectively. The six simulations are simulated at generalised Reynolds number of 6,500-9,300. Although simulations are undertaken in a Cartesian coordinate system, all results are presented in a cylindrical coordinate system in which the axial velocity is denoted by $\mathrm{U}$, the radial velocity by $\mathrm{V}$ and the azimuthal velocity by $\mathrm{W}$.

\section{$5.1 \quad$ Increased yield stress}

\subsubsection{Mean flow profile for Herschel-Bulkley fluids}

The mean axial velocity for these three simulations for $\tau_{y}=2.698,2.923,3.374$ are shown in Figure 5. They are plotted with conventional 'law of the wall' non-dimensionalisation and are compared to values obtained for the control fluid yield stress profile produced with the same DNS code. As yield stress $\tau_{y}$ increases, the profiles for the Herschel-Bulkley fluids are moving away from the logarithmic profile obtained by theoretical analysis. All three simulations plus the control simulation show indications of a log-layer profile with a greater slope than the theoretical logarithmic profile. The results for $\tau_{y}=3.374$ fall sufficiently above the theoretical profile. 


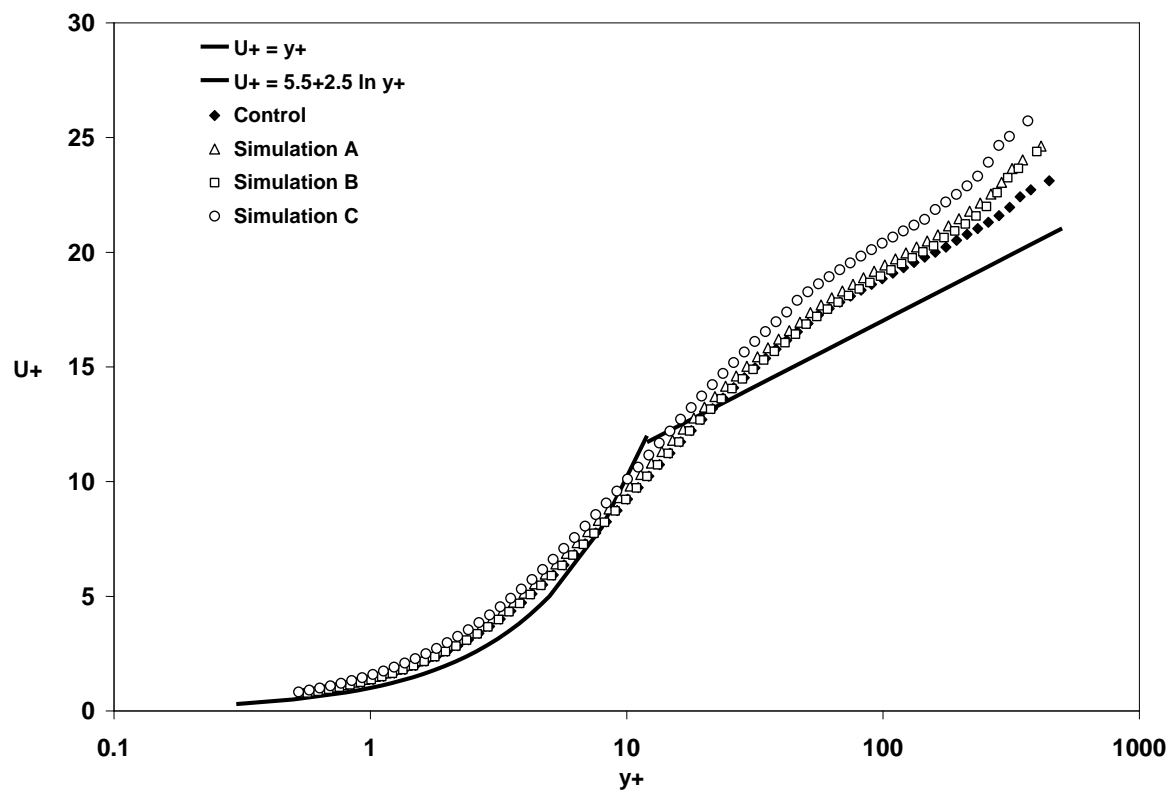

Figure 5 Mean axial velocity profiles for the turbulent flow of three different Herschel-Bulkley fluids. The profiles have been non-dimensionalised using the conventional nondimensionalisation with the mean wall viscosity taking the place of the Newtonian viscosity

\subsubsection{Turbulence statistics}

Only the control and Simulation C's turbulence intensities and turbulence production are plotted in Figure 6 in physical coordinates and in Figure 7 in wall units. The turbulent intensities in Simulation C and the control simulation are very similar. With azimuthal velocity and radial velocity fluctuations, both are lower than the Newtonian simulation. The axial velocity fluctuations are slightly higher than Newtonian simulation with increase of yield stress. This agrees with the Rudman et al. (2004) result. The differences between the control simulation and Simulation C are insignificant, as all the axial turbulence intensities are close together.

Low and high Reynolds number flows produce almost identical axial, radial and azimuthal velocity fluctuations. However, from Rudman and Blackburn (2006), it is discovered that the presence of a yield stress has the effect of reducing the cross-stream intensities more. From Figures 6 and 7, it is clearly to see that the azimuthal velocity fluctuations actually increased slightly with the increase of yield stress. It could be a result of not fixing Reynolds number to a certain value. In theory, with increased yield stress, the flows should be less developed in the channel.

The production of turbulence is given by:

$$
P_{z r}=\overline{U^{\prime} V^{\prime}} \frac{\partial \bar{U}}{\partial r}
$$

and it is plotted in Figures 6(d) and 7(d). From Figure 7(d), the maximum production occurs at a value of $y^{+} \approx 6.5$ for these simulations. In Rudman et al. (2004) it was concluded that the maximum production in wall unit is decreasing slightly with a decreased $\mathrm{n}$ value. In their case, the maximum turbulent production occurs at $y^{+} \approx 10$. From Figure 7 , Simulation $\mathrm{C}$ has shifted the maximum to a slightly smaller $y^{+}$value. 

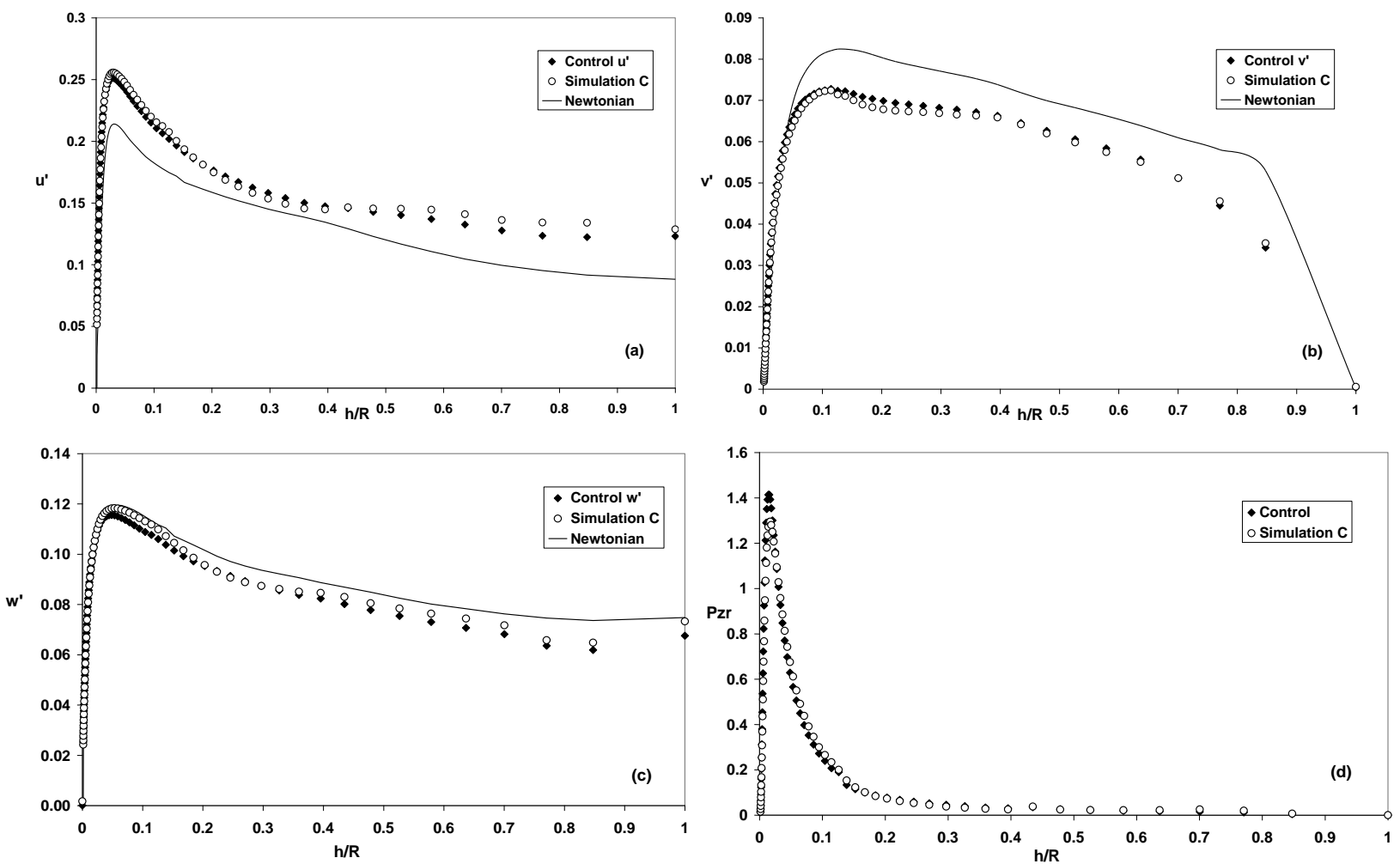

Figure 6 Turbulence intensities (a) axial, (b) radial, (c) azimuthal and (d) turbulence production plotted as a function of $h / R$ (solid line for Newtonian DNS)
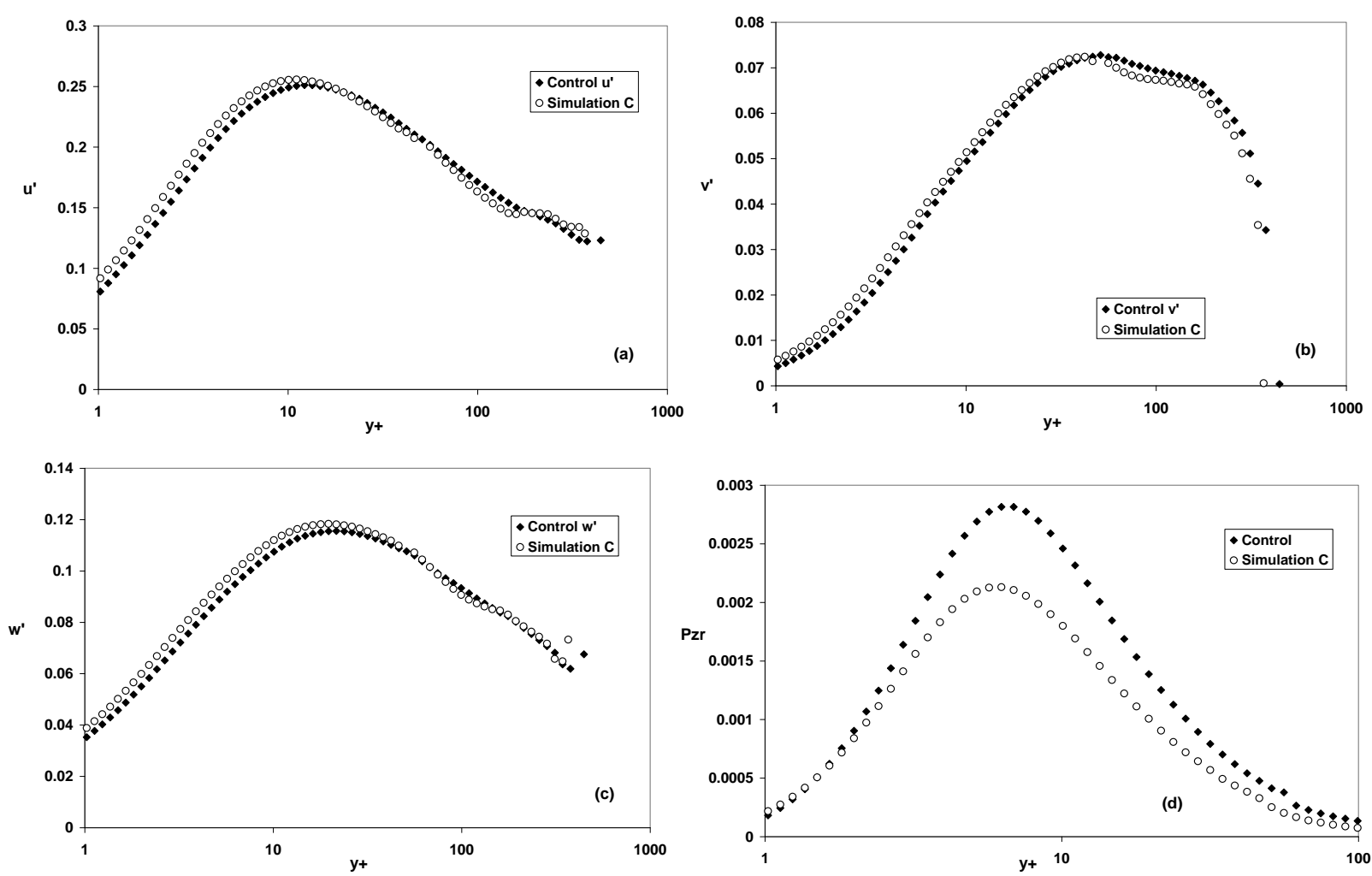

Figure 7 Turbulence intensities (a) axial, (b) radial, (c) azimuthal and (d) turbulence production plotted in wall coordinates 


\subsection{Decreased yield stress}

\subsubsection{Mean flow profile for Herschel-Bulkley fluids}

The mean axial velocity for these three simulations for $\tau_{y}=1.799,1.574,1.125$ are shown in Figure 8 . They are plotted with conventional 'law of the wall' non-dimensionalisation and are compared to control yield stress profile. As yield stress $\tau_{y}$ decreases, the profiles for the Herschel-Bulkley fluids are moving closer to the theoretical logarithmic profile, as expected.

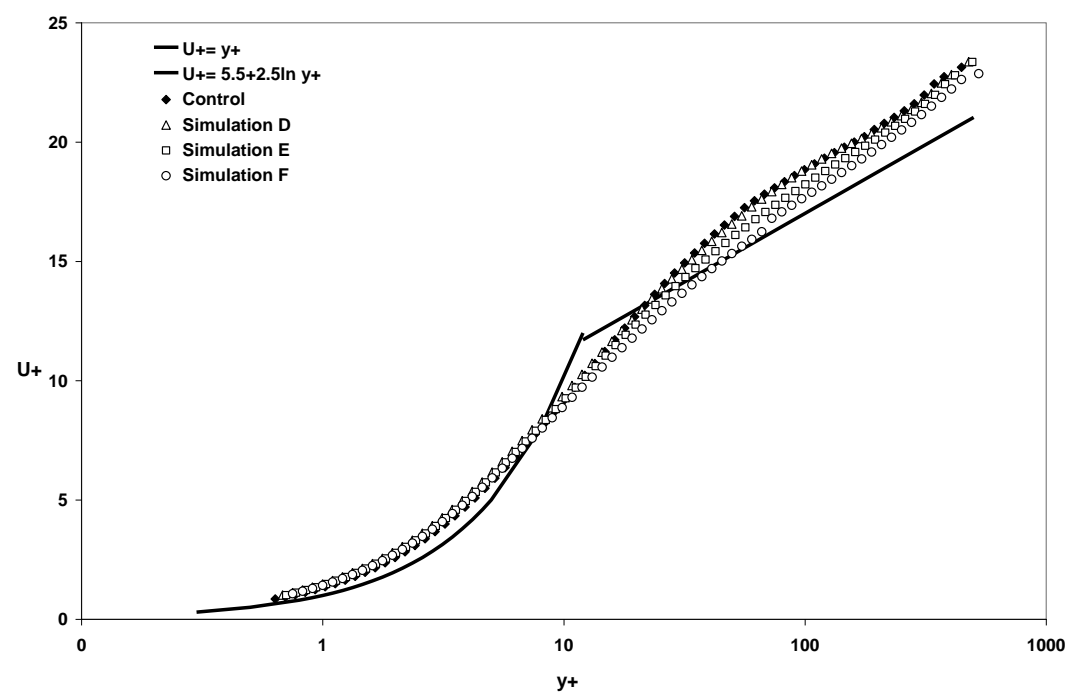

Figure 8 Mean axial velocity profiles for the turbulent flow of three different Herschel-Bulkley fluids. The profiles have been non-dimensionalised using the conventional nondimensionalisation with the mean wall viscosity taking the place of the Newtonian viscosity

\subsubsection{Turbulence statistics}

Turbulence intensities and turbulence production are plotted in Figure 9 in physical coordinates and in Figure 10, in wall units. For axial velocity fluctuations, the decreased yield stress simulations and the control simulations are very similar. Moser et al. (1999) have done direct numerical simulation for turbulent channel flow with $\operatorname{Re}_{\tau}=590$ (where as $\operatorname{Re}_{\tau}$ is around 900 for this simulation). The authors discovered that with an increase in Reynolds number, which in their case is from $180-500$, the peak of $u^{\prime}$ increased by around $4.5 \%$. Also, the u' profile appears to be the same to the high Reynolds number for $y^{+}>80$ in Moser et al. (1999). In Figures 9 and 10, the u' profile is shifted slightly to the left. Despite the shift, the shape and magnitude of the profile is quite similar to the control simulation. With azimuthal velocity and radial velocity fluctuations, both are quite higher than control simulation. In radial velocity fluctuation, Simulation $\mathrm{F}$ is closely approaching Newtonian case, especially in the wall regions. Interestingly, with a decreased yield stress, the axial velocity fluctuations are lower than control simulation but closer to Newtonian case.

From both turbulent production plots, the maximum production occurs at a value of $y^{+} \approx 6-6.5$. From Figure 10 , the simulation with smaller yield stress has higher turbulent production values than the control simulation. Simulation $\mathrm{F}$ has maximum turbulent production at similar $\mathrm{y}^{+}$value. However, Simulation $\mathrm{F}$ has shifted the maximum to a slightly smaller $\mathrm{y}^{+}$value and its peak turbulent production value. 

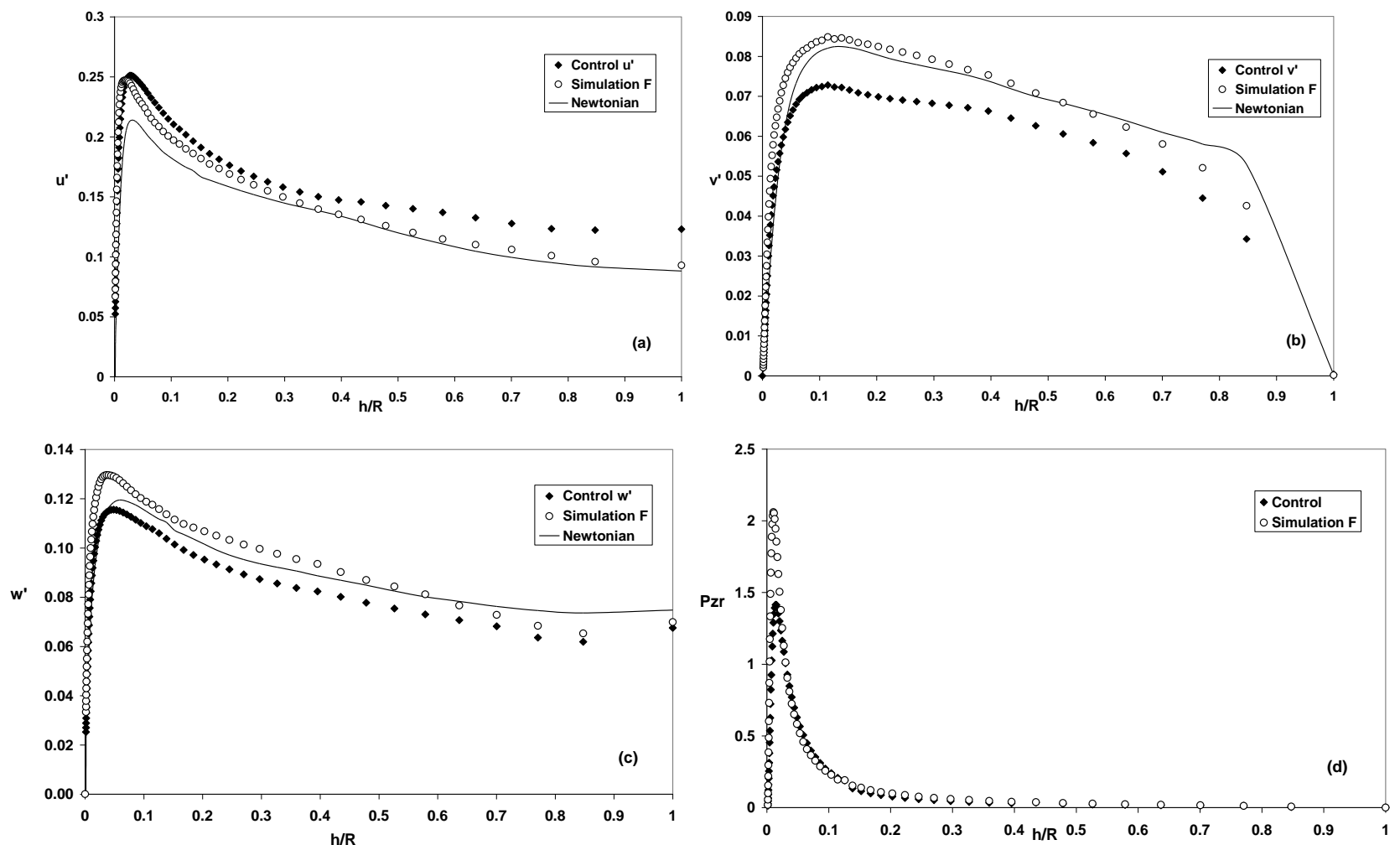

Figure 9 Turbulence intensities (a) axial, (b) radial, (c) azimuthal and (d) turbulence production plotted as a function of $h / R$ (solid line for Newtonian DNS)
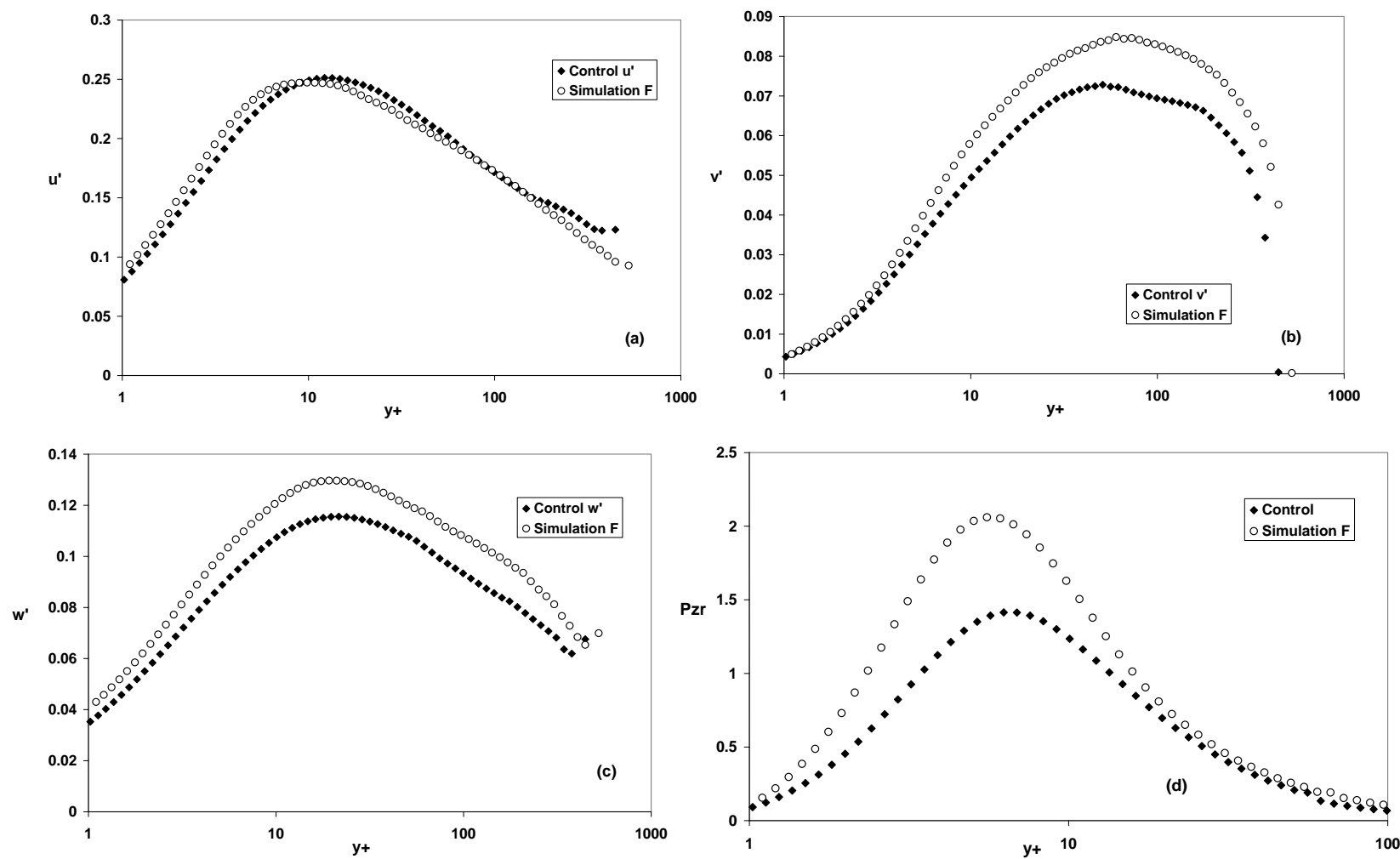

Figure 10 Turbulence intensities (a) axial, (b) radial, (c) azimuthal and (d) turbulence production plotted in wall coordinates 


\subsection{Changing in flow behaviour index $-n$}

\subsubsection{Mean flow profile for Herschel-Bulkley fluids}

The mean axial velocity for $n=0.79$ is shown in Figure 11. It is also plotted with conventional 'law of the wall' non-dimensionalisation and is compared to control flow behaviour index profile using the DNS code. It is interesting to see that the velocity profile deviates upward between $\mathrm{U}^{+}$and $y^{+}$in the near wall region. In the logarithmic region, $n=0.79$ profile is above the control profile as well as theoretical logarithmic profile. This is consistent with results presented in Pinho and Whitelaw (1989) for different concentration of CMC solutions. It was discovered that with the increased concentration of the polymer, $\mathrm{U}^{+}$value approached to the Virk's ultimate profile. However, in Pinho and Whitelaw (1989), they did not comment on the near wall region of the velocity profile. It is still unknown why the velocity profile deviates from the theoretical profile in the near wall region.

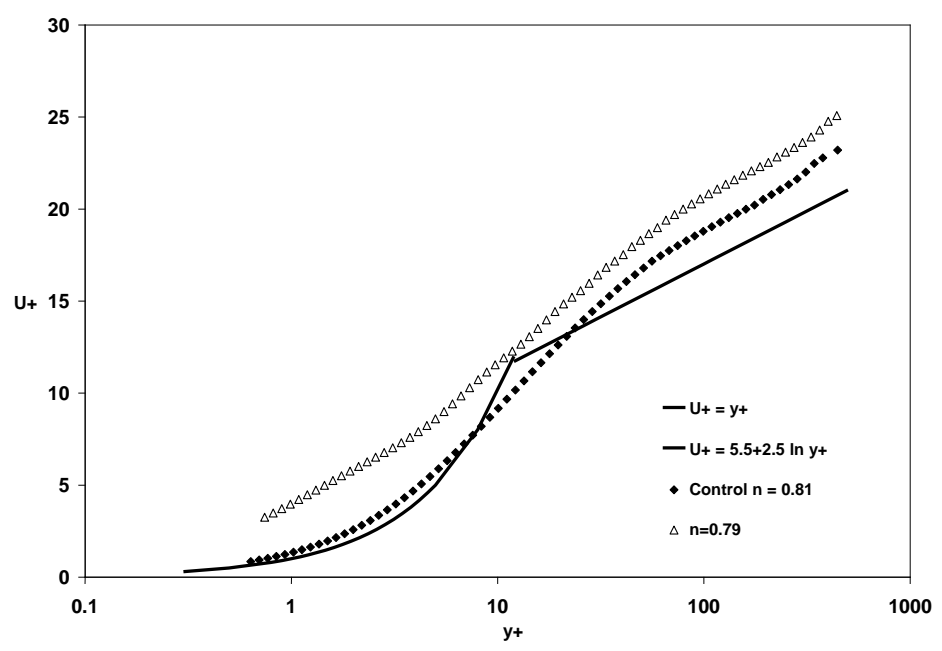

Figure 11 Mean axial velocity profile for the turbulent flow of $\boldsymbol{n}=\mathbf{0 . 7 9}$. The profile has been nondimensionalised using the conventional non-dimensionalisation with the mean wall viscosity taking the place of the Newtonian viscosity

The mean axial velocity for $n=0.90$ is shown in Figure 12. As the flow behaviour index $n$ increases, the profile for the Herschel-Bulkley fluids shifted upward from the logarithmic profile obtained by theoretical analysis. The results for $n=0.90$ fall sufficiently above control simulation as well as the theoretical profile. Rudman et al. (2001) showed similar result with weakly turbulent flow of a power law pipe flow. It was concluded that the mean flow profile approaches the theoretical profile as Reynolds number increases. In Rudman et al. (2001) paper, the $\mathrm{Re}=3,964$ velocity profile also fell above their control simulation $\operatorname{Re}=5,500$. In this case, the Reynolds number for $n=0.90$ simulation is 4,636 which considerably smaller than the control simulation i.e. 7,800.

Clapp (1961), proposed a velocity distribution equation for power law fluid which included an equation for viscous sub layer, buffer sub layer and turbulent layer.

Viscous sub-layer:

$$
U^{+}=\left(y^{+}\right)^{1 / n}, 0<y^{+}<5^{n}
$$

Buffer sub-layer:

$$
U^{+}=\frac{5}{n} \ln y^{+}-3.05,5^{n}<y^{+}<y_{2}^{+}
$$


Turbulent layer:

$$
U^{+}=\frac{G}{n} \ln y^{+}+H, y^{+}>y^{+}{ }_{2}
$$

$G$ and $H$ are empirical constants to be obtained by experiment. The observed velocity distributions by the author were correlated by the equation:

$$
U^{+}=\frac{2.78}{n} \ln y^{+}+\frac{3.8}{n}
$$

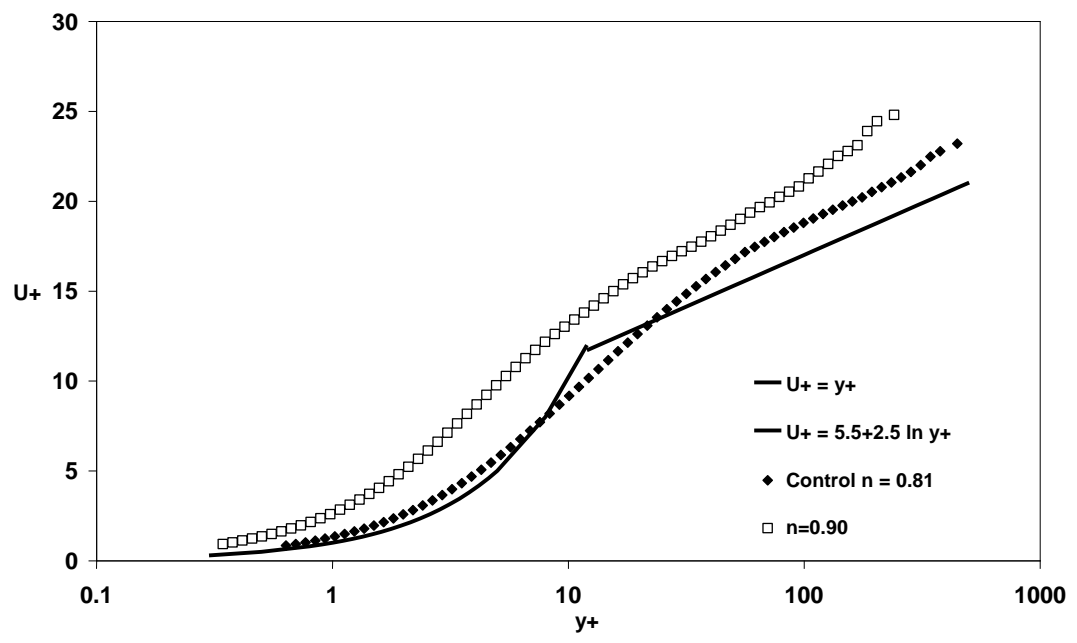

Figure 12 Mean axial velocity profile for the turbulent flow of $\boldsymbol{n}=\mathbf{0 . 9 0}$. The profile has been nondimensionalised using the conventional non-dimensionalisation with the mean wall viscosity taking the place of the Newtonian viscosity

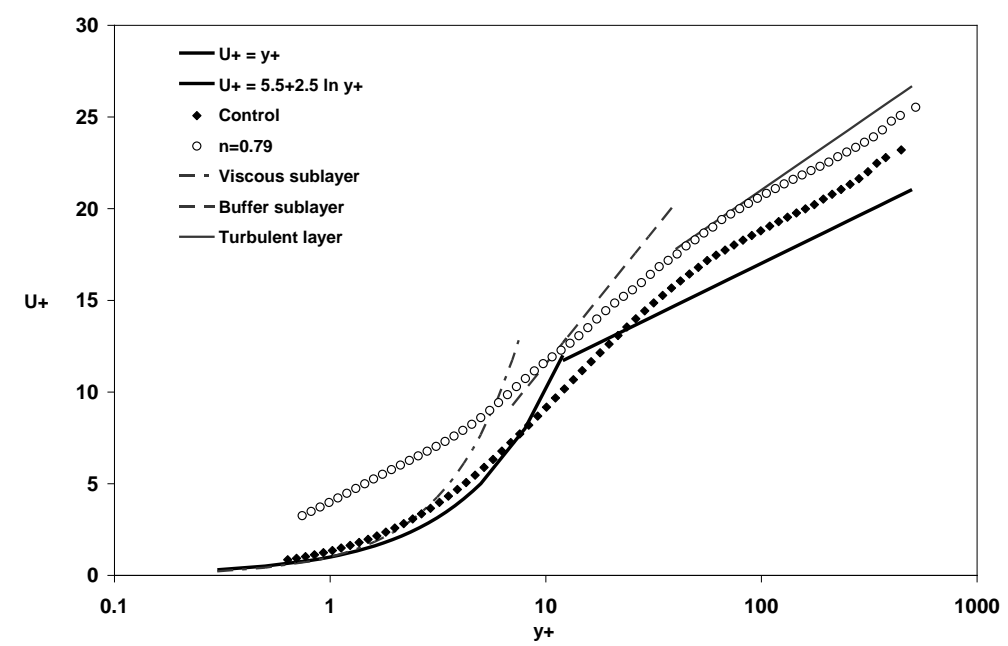

Figure 13 Clapp's (1961) velocity correlation and mean axial velocity profile for the turbulent flow of $n=0.79$

It is interesting to see whether the inclusion of fluid index $n$ can allow a better fit for the velocity distribution equation. In Figure 13, Clapp's (1961) velocity distribution is plotted with the mean axial velocity profile for $n=0.79$. With Clapp's velocity distribution, it is quite clear that turbulent layer and buffer sub layer suit the simulation axial velocity quite well. However, for viscous sub layer, the velocity profile for simulation is still deviated from the logarithmic law as well as the Clapp's velocity distribution. 
The simulated bulk velocity for $n=0.79$ and $n=0.90$ is $1.61 \mathrm{~m} / \mathrm{s}$ and $1.54 \mathrm{~m} / \mathrm{s}$ respectively. These velocities are higher than the experimental value. However, with $n=0.79$ and $n=0.90$ both showed shifting velocity profile upward in the above comparison, it is still very difficult to see the real effect of flow behaviour index to the simulation. In future investigation, the Reynolds number will be fixed in order to examine the full effect of flow behaviour index.

\section{Conclusion}

The simulation results for a Herschel-Bulkley fluid show some agreement as well as some significant differences with the experimental results. A comparison between the experimentally measured velocity profile in an open channel and the simulation data indicates some discrepancies between the results obtained. The study of yield stress is being undertaken to address this issue. The mean flow profile deviates further from the Newtonian profile as a yield stress increases. Therefore it is concluded that the flow will be better developed with a decreased yield stress. Furthermore, Clapp's (1961) velocity distribution equation can capture the effect of flow behaviour index quite well. With a decrease of flow behaviour index, the buffer sub-layer and turbulent layer fit particular well with Clapp's distribution equation. However, yield stress effect and flow behaviour index effect could not fully explain the velocity discrepancy between experimental data and simulation data, future work is needed to investigate the last rheological parameter: K. The inability of capturing secondary flow by the simulation code is not discussed here. The application of DNS to flows of non-Newtonian fluids can provide an improved understanding of the effect of rheological parameters on turbulent flow.

\section{Acknowledgements}

The authors gratefully acknowledge the support of the ARC for scholarship of the work described in this paper.

\section{References}

Clapp, R.M. (1961) Turbulent heat transfer in pseudoplastic non-Newtonian fluids, International Development of Heat Transfer, Part III, New York.

Dimitropoulos, C., Dubief, Y., Shaqfeh, E., Moin, P. and Lele, S. (2005) Direct numerical simulation of polymerinduced drag reduction in turbulent boundary layer flow, Physics of Fluids, Vol. 17, 011705.

Fitton, T. (2007) Tailings beach slope prediction, PhD thesis, RMIT University, Melbourne, Australia.

Joung, Y., Choi, S.U. and Choi, J.I. (2007) Direct numerical simulation of turbulent flow in a square duct: analysis of secondary flows, Journal of Engineering Mechanics, Vol. 133(2), pp. 213-221.

Kim, J., Moin, P. and Moser, R. (1987) Turbulence statistics in fully developed channel flow at low Reynolds number, Journal of Fluid Mechanics, Vol. 177, p. 133.

Moser, R., Kim, J. and Mansour, N. (1999) Direct numerical simulation of turbulent channel flow up to Re $\tau=590$, Physics of Fluids, Vol. 111(4), pp. 943-945.

Nezu, I. (2005) Open-Channel flow turbulence and its research prospect in the 21st century, Journal of Hydraulic Engineering, Vol. 31(4), pp. 229-246.

Nezu, I. and Nagakawa, H. (1993) Turbulence in Open Channel Flows, IAHR AIRH, Netherlands.

Pinho, F. and Whitelaw, J. (1989) Flow of Non-Newtonian fluids in a pipe, Journal of Non-Newtonian Fluid Mechanics, Vol. 34(1990), pp. 129-144.

Rudman, M. and Blackburn, H.M. (2003) The effect of shear thinning behaviour on turbulent pipe flow, Third International Conference on CFD in the Minerals and Process Industries, CSIRO, Melbourne, Australia.

Rudman, M. and Blackburn, H.M. (2006) Direct numerical simulation of turbulent non-Newtonian flow using a spectral element method, Applied Mathematical Modelling, Vol. 130, pp. 1229-1248.

Rudman, M., Blackburn, H.M., Graham, L.J. and Pullum, L. (2001) Weakly turbulent pipe flow of a power law fluid, 14th Australasian Fluid Mechanics Conference, Adelaide University, Adelaide, Australia.

Rudman, M., Blackburn, H.M., Graham, L.J. and Pullum, L. (2004) Turbulent pipe flow of shear-thinning fluids, Journal Non-Newtonian Fluid Mechanics, Vol. 118, pp. 33-48.

Sureskumar, R., Beris, A. and Handler, A. (1997) Direct numerical simulation of the turbulent channel flow of a polymer solution, Physics of Fluids, Vol. 9, pp. 743-755.

Verbanck, M. (2000) Computing near-bed solids transport in sewers and similar sediment-carrying open-channel flows, Urban Water, Vol. 2, pp. 277-284. 
Wilson, K.C. (1991) Slurry transport in flumes, Slurry handling design of solid liquid system, N.P. Brown, N.I. Heywood (eds), Elsevier, London. 